\title{
BMJ Open Sipjeondaebo-tang in patients with breast cancer with fatigue: a protocol for a pilot, randomised, double-blind, placebo-controlled, cross-over trial
}

\author{
Chunhoo Cheon, ${ }^{1}$ Sohyeon Kang, ${ }^{1}$ Youme Ko, ${ }^{1}$ Mia Kim, ${ }^{2}$ Bo-Hyoung Jang, ${ }^{1}$ \\ Yong-Cheol Shin, ${ }^{1}$ Seong-Gyu Ko ${ }^{1}$
}

To cite: Cheon C, Kang S, Ko Y, et al. Sipjeondaebo-tang in patients with breast cancer with fatigue: a protocol for a pilot, randomised, doubleblind, placebo-controlled, cross-over trial. BMJ Open 2018;8:e021242. doi:10.1136/ bmjopen-2017-021242

- Prepublication history for this paper is available online. To view these files, please visit the journal online (http://dx.doi. org/10.1136/bmjopen-2017021242).

Received 19 December 2017 Revised 4 May 2018 Accepted 14 May 2018

\section{ABSTRACT}

Introduction Cancer-related fatigue is a frequent symptom in patients with cancer and one of the most distressing symptoms in patients with breast cancer. Sipjeondaebo-tang (Juzen-taiho-to in Japanese or ShiQuan-Da-Bu-Tang in Chinese) is a widely used herbal medicine for the treatment of fatigue in Korea, China and Japan. The purpose of the present study is to evaluate the feasibility of Sipjeondaebo-tang for cancer-related fatigue. Methods and analysis The present study is a randomised, double-blind, placebo-controlled, cross-over study. Forty-eight patients with breast cancer who are indicated for doxorubicin and cyclophosphamide will be recruited. The participants will receive $3 \mathrm{~g}$ of Sipjeondaebotang or a placebo three times a day for 56 days. The primary outcome measurement is the change in the Brief Fatigue Inventory scores. The secondary outcome measurements include the changes in the Visual Analogue Scale (VAS) of fatigue, and quality of life measured by the European Organization for Research and Treatment of Cancer-QLQ-C30 and QLQ-BR23. VAS of fatigue will be measured on every visit, and other outcomes will be measured on visits 2, 4,6 and 7. The total study period is 14 weeks.

Ethics and dissemination This study has been approved by the Institutional Review Board of the Catholic Kwandong University International St Mary's Hospital (reference IS16MNSI0011). The results of this study will be published in a peer-reviewed journal and presented at a scientific conference.

Trial registration number NCT02858856; Pre-results.

\section{INTRODUCTION}

Cancer-related fatigue is a frequently experienced symptom in patients with cancer regardless of tumour type, and one of the most distressing symptoms in patients with breast cancer. ${ }^{1}$ The National Comprehensive Cancer Network defined cancer-related fatigue as a distressing, persistent, subjective sense of physical, emotional and/or cognitive tiredness or exhaustion related to cancer or cancer treatment that is not proportionate to recent activity. ${ }^{2}$ Fatigue is the most commonly
Strengths and limitations of this study

- This study is the first randomised controlled trial to evaluate the feasibility of Sipjeondaebo-tang for the treatment of cancer-related fatigue.

- All participants receive identical chemotherapy regimens.

- The limitations of this study are its relatively small number of participants and the fact that it will be conducted in a single institution.

reported side effect of chemotherapy, alongside pain, nausea and vomiting. ${ }^{3}$ In patients with breast cancer receiving chemotherapy, the prevalence of fatigue is greater than $72 \% .{ }^{45}$ In a study conducted at a hospital in South Korea, the prevalence of fatigue was $32.3 \%$ among patients with cancer and $45.5 \%$ among female patients with cancer. ${ }^{6}$

Managing cancer-related fatigue is one of the main concerns in the management of patients with cancer. Cancer-related fatigue leads to considerable socioeconomic costs for patients with cancer. Patients with cancer face difficulties in their daily lives due to fatigue, and they miss an average of 4.2 days of work per month. The primary caregivers of patients with cancer are also negatively influenced, missing an average of 4.5 days of work per month. ${ }^{7}$ Cancer-related fatigue also compromises the quality of life and, in severe cases, it negatively affects the effectiveness of treatment by prompting dose reductions, delaying anticancer therapy or reducing adherence to prescribed drugs. ${ }^{8}$ Therefore, management of cancer-related fatigue is very important.

Several factors contribute to the development of cancer-related fatigue. However, the pathological mechanisms involved are not well known. ${ }^{9}$ Currently, the underlying pathologies of cancer-related fatigue 
are treated with haematopoietic agents or antidepressants, but the treatment is not effective against fatigue produced by other aetiologies, or it only improves anaemia or depression. ${ }^{10}$ Furthermore, cancer-related fatigue is not treated adequately to meet the needs of patients with cancer. More than half $(61 \%)$ of the patients with cancer answered that fatigue has greater influence on their lives than pain; however, only $27 \%$ of patients with cancer received specific recommendations for fatigue treatment. There is no definitive treatment for cancer-related fatigue, so only $9 \%$ of physicians prescribe drugs to treat fatigue. ${ }^{7}$ Therefore, more research on evidence-based interventions for cancer-related fatigue is needed.

Sipjeondaebo-tang (SJDBT; Juzen-taiho-to in Japanese or Shi-Quan-Da-Bu-Tang in Chinese) is a widely used herbal medicine in Korea, China and Japan. In Korea, it is the third most commonly prescribed herbal medicine. ${ }^{11}$ According to Korean medicine theory, SJDBT treats the syndrome of dual deficiency of qi and blood by balancing Yin and Yang. ${ }^{12}$ The Ministry of Food and Drug Safety (MFDS) has approved SJDBT to treat weakness after illness, anorexia, night sweats, cold hands and feet, and anaemia. ${ }^{13}$ Despite the frequent use of SJDBT for the treatment of fatigue, there have only been clinical trials on haematotoxicity ${ }^{14}$ and anaemia, ${ }^{15}{ }^{16}$ and scientific evidence for SJDBT on cancer-related fatigue is lacking. Therefore, the aim of the present study is to evaluate the feasibility of SJDBT for fatigue in patients with breast cancer receiving chemotherapy. To achieve this aim, a randomised, double-blind, placebo-controlled, cross-over trial has been planned.

\section{METHODS AND ANALYSIS \\ Study design}

A randomised, double-blind, placebo-controlled, crossover trial will be conducted at the Catholic Kwandong University International St Mary's Hospital in Incheon, Republic of Korea. Any participants meeting the eligibility criteria will be enrolled. After enrolment, the participants will be randomly allocated to two groups: group A and group B. A schematic flow of the study is shown in figure 1. Both groups will receive four cycles of doxorubicin and cyclophosphamide chemotherapy, and each cycle will last 21 days. There is no expected protocol modification. However, if it happens, any modification in the protocol will be communicated to the investigators via conference calls. The final manuscript for publication will include all the amendments.

\section{Recruitment}

Patients who visit the trial institution and fulfil the eligibility criteria will be invited to participate in the study by the investigator in charge. The investigators will provide detailed trial information including study period, purpose, eligibility criteria, intervention and design.

\section{Participants}

\section{Inclusion criteria}

Men and women aged 20-65years, patients who have histologically or cytologically confirmed breast cancer, patients who are indicated for doxorubicin and cyclophosphamide, Eastern Cooperative Oncology Group performance status score $0-2$ and patients willing and able to give informed consent for participation in the study

\section{Exclusion criteria}

Patients who are unable to take drugs orally; patients receiving neoadjuvant chemotherapy; patients with mental illness such as dementia, delirium and depression; patients with hepatitis B, hepatitis C or liver cirrhosis; patients with severe renal disability (two times higher than the upper limit of normal for serum creatinine); patients with severe liver disability (three times higher than the upper limit of normal for alanine transaminase and aspartate transaminase); patients with diabetes (haemoglobin A1c $>8 \%$ ) or hypertension (systolic blood pressure $>160 \mathrm{~mm} \mathrm{Hg}$ or diastolic blood pressure $>100 \mathrm{~mm} \mathrm{Hg}$ ) that is not controlled by diet or medication; patients with thyroid disease; patients with severe systemic disease; use of other investigational products within 30 days of the study period; patients with known prior hypersensitivity to the investigational product and individuals who are judged inappropriate for the study.

\section{Subject withdrawal criteria}

The participants who meet the following criteria will be discontinued from the study: the occurrence of a serious adverse event related to investigational product, investigator's decision that it is not appropriate to proceed the trial, participants who do not comply with investigator's instruction, occurrence of significant protocol violations and participant's withdrawal of consent or request to stop taking medication. The participants who are withdrawn from the study after allocation will be followed for outcomes as far as possible.

\section{Sample size}

This is a pilot study that examines the feasibility of conducting a large-scaled randomised clinical trial of SJDBT for treating cancer-related fatigue in patients with breast cancer. Considering that a sample size between 24 and 50 has been recommended for a pilot study, ${ }^{18}$ and the sample size of other similar pilot studies, a total of 48 participants will be recruited for the present study. ${ }^{19} 20$ Thus, 24 participants will be allocated to group A and another 24 to group B.

\section{Randomisation and allocation}

The participants who meet the inclusion and exclusion criteria will be randomly allocated using random numbers generated by the Contract Research Organisation, Institute of Safety, Efficacy and Effectiveness Evaluation for Korean Medicine (ISEE). Block randomisation using R software with block size of four will be conducted. 


\section{Enrolment}

Allocation

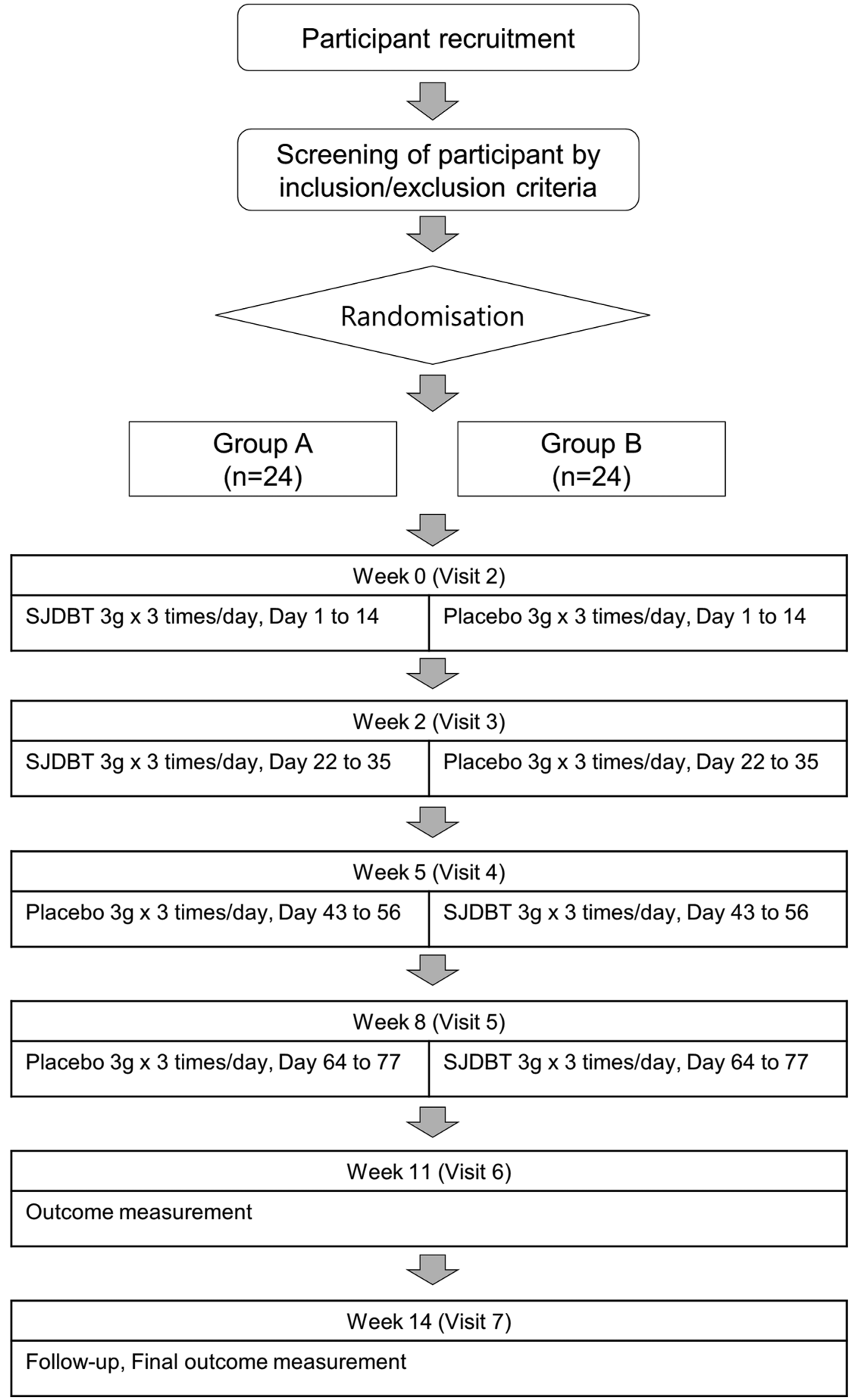

Figure 1 Study flow chart. SJDBT, Sipjeondaebo-tang.

The enrolled participants will be assigned to one of two groups with the allocation ratio of 1:1. The randomisation table will be kept in an opaque and sealed envelope by the ISEE, and it will be unclosed according to standard operating procedures (SOPs).

\section{Blinding}

A researcher at ISEE will prepare computer-generated random numbers and a randomisation table. Hanpoong Pharm and Foods Co will produce and label the investigational product in accordance with Korea Good
Manufacturing Practice standards. The labelled investigational product will be provided to the study institution by the pharmaceutical company. Since ISEE is an independent centre from the trial institution, investigators involved in recruitment, treatment and outcome assessment will be blinded to the allocation.

\section{Treatment protocol}

The participants will receive SJDBT or placebo for a total of 56 days. They will take $3 \mathrm{~g}$ of the investigational product orally with water three times a day after meals. 
The administration periods of the investigational product will be 2 weeks prior to the visiting day for chemotherapy. The participants of group A will take SJDBT from day 1 to day 14 and from day 22 to day 35 , and placebo from day 43 to day 56 and from day 64 to day 77 of the trial period. The participants of group B will take placebo from day 1 to day 14 and from day 22 to day 35, and SJDBT from day 43 to day 56 and from day 64 to day 77 of the trial period. The participants will be asked to return the drug remains for the sake of calculating compliance. During the clinical trial, the participants will be prohibited to get other treatment for fatigue.

\section{Interventions}

SJDBT is an herbal medicine that has been approved by the MFDS. It consists of $1 \mathrm{~g}$ Cinnamomi Cortex, $1 \mathrm{~g}$ Paeoniae Radix, $1 \mathrm{~g}$ Atractylodis Rhizoma Alba, $1 \mathrm{~g}$ Ginseng Radix Alba, $1 \mathrm{~g}$ Cnidii Rhizoma, $1 \mathrm{~g}$ Astragali Radix, $1 \mathrm{~g}$ Poria Sclerotium, $1 \mathrm{~g}$ Rehmanniae Radix Preparata, $1 \mathrm{~g}$ Angelicae Gigantis Radix and $0.5 \mathrm{~g}$ Glycyrrhizae Radix. These raw materials, together with lactose hydrate and corn starch, will be concentrated to a single dose of $3 \mathrm{~g}$. The placebo consists of lactose, corn starch and caramel colouring, and it has an appearance, weight, colour and taste similar to those of SJDBT. The investigational product used in the present study is a dark brown-coloured granule. The present study is an investigator initiated trial funded by the government, and the role of pharmaceutical company is limited to provide the investigational product.

\section{Primary outcome measurement}

The primary outcome is the change in the usual fatigue severity of the Brief Fatigue Inventory (BFI) between the duration of the SJDBT administration and the duration of placebo administration. BFI is an instrument for the rapid assessment of subjective fatigue status in patients with cancer. Its reliability and sensitivity has been validated. ${ }^{21}$ The development of the Korean version of the BFI was led by the National Cancer Center in Korea. The Korean version of the BFI has now been validated. ${ }^{22}$ The usual fatigue severity of BFI has been validated as a sensitive and reliable clinical indicator in Korean patients with cancer, ${ }^{23}$ and the clinical implication of the worst fatigue severity of BFI has also been validated. ${ }^{21}$ The BFI consists of nine items on an 11-point rating scale and can be measured within $10 \mathrm{~min}$. The BFI will be measured by a trained researcher at visit 2, 4, 6 and 7 according to SOPs.

\section{Secondary outcome measurements}

Secondary outcome measurements include the global BFI score, worst fatigue severity of BFI, changes in the Visual Analogue Scale (VAS) of fatigue and quality of life measured by the European Organization for Research and Treatment of Cancer (EORTC)-QLQ-C30 and QLQ-BR23. EORTC-QLQ-C30 is a questionnaire developed to evaluate the quality of life in patients with cancer and QLQ-BR23 is a breast cancer-specific module. ${ }^{24}$
Although some limitations have been reported, three fatigue questions on EORTC-QLQ-C30 have been independently validated as a measure of fatigue. ${ }^{25} 26$ The Korean version of the EORTC-QLQ-C30 and QLQ-BR23 has now been validated in Korean patients with cancer. ${ }^{27} 28$ The study schedule according to The Standard Protocol Items: Recommendations for Interventional Trials is detailed in table 1.

\section{Safety outcomes}

All safety-related variables including vital signs, physical examination, haematological test, biochemical test, urine test and adverse events will be recorded on the case report form (CRF) at every visit. Haemoglobin, haematocrit, red blood cell indices, white blood cell differential count, platelet and so on will be measured in the blood, and nitrite, albumin, bilirubin, ketone, protein and so on will be measured in the urine. Adverse events will be evaluated using the National Cancer Institute (Bethesda, Maryland, USA) Common Terminology Criteria for Adverse Events V.4.03 ${ }^{29}$ The adverse events will be assessed by a trained investigator at every visit and if any participant wishes to consult a doctor for any reason, including the occurrence of an adverse event, they can contact the investigator in charge at any time, or visit the trial institution for examination, and all of this will be recorded on the CRF. A serious adverse event will be considered to be an event with the following outcomes: death, life-threatening condition, hospitalisation or prolongation of hospitalisation, disability or permanent damage.

\section{Statistical analysis}

\section{Efficacy assessment}

The baseline characteristics will be analysed by an independent two-sample t-test for continuous variables or the $\chi^{2}$ test for the categorical variables (Fisher's exact test will be used when the expected value is $<5$ ). Alternatively, if the normality assumption is not satisfied, Wilcoxon rank-sum test will be conducted for the continuous variables. The normality assumption will be assessed by the Shapiro-Wilk test. The continuous variables will be presented as the mean $\pm \mathrm{SD}$ or median and range, and the categorical variables will be presented as the $\mathrm{n}(\%)$ or the absolute and relative frequencies. Statistical analyses for efficacy will be conducted for both ITT (intention-to-treat, all randomised participants who receive at least one dose of the study drug) and PP (per-protocol, a subset of the participants who complete the study without any major protocol deviations) data sets. ITT analyses will be considered as primary analyses, and PP analysis will be considered as secondary analysis. The missing values will be imputed by multiple imputation. For the primary outcome measure, the mean differences of BFI between the SJDBT administration period and the placebo administration period will be compared using an independent two-sample t-test. If the normal distribution assumption is not satisfied for the continuous variables, Wilcoxon 
Table 1 Study schedule of the SJDBT trial (14 weeks)

\begin{tabular}{|c|c|c|c|c|c|c|c|c|}
\hline \multirow{3}{*}{\multicolumn{2}{|c|}{ Timepoint }} & \multicolumn{7}{|c|}{ Study period } \\
\hline & & \multirow{2}{*}{$\begin{array}{l}\text { Enrolment } \\
\text { Day }-7 \\
\text { (V1) }\end{array}$} & \multirow{2}{*}{$\begin{array}{l}\text { Allocation } \\
\text { Day } 0 \\
\text { (V2) }\end{array}$} & \multicolumn{4}{|c|}{ Postallocation } & \multirow{2}{*}{$\begin{array}{l}\text { Close-out } \\
\text { Day } 98 \\
\text { (V7) }\end{array}$} \\
\hline & & & & $\begin{array}{l}\text { Day } 14 \\
\text { (V3) }\end{array}$ & $\begin{array}{l}\text { Day } 35 \\
\text { (V4) }\end{array}$ & $\begin{array}{l}\text { Day } 56 \\
\text { (V5) }\end{array}$ & $\begin{array}{l}\text { Day } 77 \\
\text { (V6) }\end{array}$ & \\
\hline \multicolumn{2}{|c|}{ Eligibility screen } & $x$ & & & & & & \\
\hline \multicolumn{2}{|c|}{ Informed consent } & $x$ & & & & & & \\
\hline \multicolumn{2}{|c|}{ Allocation } & & $x$ & & & & & \\
\hline \multirow[t]{2}{*}{ Group A } & SJDBT & & $\mathrm{X}^{*}$ & $X^{*}$ & & & & \\
\hline & Placebo & & & & $X^{*}$ & $X^{*}$ & & \\
\hline \multirow[t]{2}{*}{ Group B } & SJDBT & & & & $X^{*}$ & $X^{*}$ & & \\
\hline & Placebo & & $X^{*}$ & $X^{*}$ & & & & \\
\hline \multicolumn{2}{|c|}{ Demographic characteristic } & $\mathrm{x}$ & & & & & & \\
\hline \multicolumn{2}{|l|}{ Vital signs } & $x$ & $x$ & $x$ & $\mathrm{x}$ & $x$ & $\mathrm{x}$ & $x$ \\
\hline \multicolumn{2}{|c|}{ Physical examination } & $x$ & $x$ & $x$ & $x$ & $x$ & $x$ & $x$ \\
\hline \multicolumn{2}{|c|}{ Laboratory test } & $x$ & $x$ & $x$ & $x$ & $x$ & $x$ & $x$ \\
\hline \multicolumn{2}{|c|}{ Brief fatigue inventory } & & $x$ & & $\mathrm{x}$ & & $x$ & $x$ \\
\hline \multicolumn{2}{|c|}{ EORTC-QLQ-C30 } & & $x$ & & $x$ & & $x$ & $x$ \\
\hline \multicolumn{2}{|c|}{ EORTC-QLQ-BR23 } & & $x$ & & $x$ & & $x$ & $x$ \\
\hline \multicolumn{2}{|c|}{ VAS for fatigue } & & $x$ & $x$ & $\mathrm{x}$ & $x$ & $\mathrm{x}$ & $x$ \\
\hline
\end{tabular}

*The administration periods of the investigational product are 2 weeks prior to each visit. (Days 1-14, days 22-35, days 43-56, days 64-77).

EORTC, European Organization for Research and Treatment of Cancer; SJDBT, Sipjeondaebo-tang; VAS, Visual Analogue Scale.

rank-sum test will be conducted. For the secondary outcome measure, the mean differences of fatigue VAS, EORTC-QLQ-C30 and QLQ-BR23 between the SJDBT administration period and the placebo administration period will be compared using an independent two-sample t-test or Wilcoxon rank-sum test. A p value of $<0.05$ will be regarded as statistically significant. The present study does not consider an interim analysis.

\section{Safety assessment}

The participants will be asked to report any adverse events that may occur during the trial at every visit. Any identified adverse event will be recorded in the CRFs. If a severe adverse event occurs and is associated with the investigational product, the participant will be withdrawn from the study and receive appropriate treatment. Any loss caused by the study will be compensated by insurance. Safety-related variables, including laboratory test results and adverse events, will be compared between the SJDBT administration period and the placebo administration period using the ITT dataset.

\section{Data and safety monitoring}

The ISEE will monitor the present study for quality control according to SOPs. The trial institution will be monitored while this study is ongoing. There is no plan for auditing. For data quality improvement, double data entry and range checks for data values will be conducted. Adverse reactions will be reported to the institutional review board, and serious and unexpected adverse reactions will be reported to regulatory authorities. There will be no coordinating centre, steering committee or endpoint adjudication committee in the present study.

\section{Ethics and dissemination}

The current protocol version is 1.3. Written informed consent will be obtained prior to study commencement from each participant by the investigator. The present study will be conducted in compliance with the Declaration of Helsinki and Korean Good Clinical Practice published by the MFDS. Any information obtained from the participants will be handled confidentially. During the entire trial period, the data will be handled by the study identification number which is assigned to each participant at enrolment. All the records from the trial will be retained secure in a locked cabinet or password-protected files. Only investigators in charge will have authority to access the data. The results of this study will be published in a scientific journal. Thus far, there is no plan for public release of the full protocol and individual datasets.

\section{Patient and public involvement}

There was no patient or public involvement in the design of the present study, and there is no planned patient or public involvement to recruit and conduct the study. 


\section{DISCUSSION}

The present study investigates the feasibility of SJDBT for cancer-related fatigue in patients with breast cancer. SJDBT is a widely used herbal medicine in Korea, China and Japan, and its pharmacological efficacy has been partly reported. In preclinical studies on cancer, SJDBT has been shown to inhibit melanoma metastasis by inducing natural killer cell activity and suppressing vascularisation, ${ }^{30} 31$ alleviate cancer-induced anorexia and cachexia, ${ }^{32}$ and protect against anticancer drug-induced myelosuppression. ${ }^{33}$ It also exhibits antiangiogenic and immunomodulatory effects in malignant glioma ${ }^{34}$ and preventive effects in endometrial carcinogenesis. ${ }^{35} \mathrm{~A}$ case report suggested that SJDBT combined with gemcitabine enhances the antitumour effects in advanced biliary tract cancer. ${ }^{36}$ Furthermore, SJDBT protects against carbon tetrachloride-induced anorexia and hepatotoxicity, ${ }^{37}$ protects the gastric mucosa by exerting antioxidant effects, ${ }^{38}$ inhibits retinal neovascularisation by inhibiting angiogenesis ${ }^{39}$ and restraint stress. ${ }^{40}$ Its potential therapeutic effect on Alzheimer's disease has also been reported. ${ }^{41}$ These studies suggest possible use of SJDBT on various diseases, remaining the need of further research. Although there are several published papers on the efficacy of SJDBT in patients with cancer, the present study has its own significance. While previous studies focused on haematotoxicity, ${ }^{14}$ quality of life, ${ }^{42}$ anorexia ${ }^{43}$ and immunity ${ }^{44}$ of patients with cancer, the present study focuses on cancer-related fatigue. The limitations of the present study include its small sample size and single-centre design. The sample size of the present study is quite small compared with that of studies that evaluated the efficacy of American ginseng for cancer-related fatigue. ${ }^{45}{ }^{46}$ However, to the best of our knowledge, this is the first randomised controlled trial to evaluate the efficacy of SJDBT for cancer-related fatigue. So far, most studies on patients with cancer using traditional Korean medicine conducted in Korea are smallscale studies. Similar to other small pilot studies on sleep disturbance,${ }^{47}$ fatigue, ${ }^{48}$ pain ${ }^{49}$ and anorexia ${ }^{50}$ in patients with cancer conducted in Korea, which have provided evidence for further study, we hope that the present study will also promote further studies and the use of herbal medicines in patients with cancer. The present study may be regarded as a study that investigates preventive effect of SJDBT in fatigue during chemotherapy. However, one of the limitations of the present study is the difficulty to define clearly whether the fatigue is due to chemotherapy, surgery or breast cancer. Based on the results of this study, a further, large-scale study to investigate the efficacy of SJDBT in patients with significant fatigue during the first cycle of chemotherapy is needed. Furthermore, the present study reduces patient heterogeneity by specifying not only cancer types but also types of chemotherapy. Besides, this study assesses quality of life to evaluate the effect of SJDBT on general health status. Even though it has a few limitations, the present study would be meaningful in that it is a pilot study to plan further large-scale trials by evaluating not only cancer-related fatigue but also quality of life due to overall symptoms.

Acknowledgements The authors wish to acknowledge Hanpoong Phar. \& Foods Co Ltd for providing investigational product support.

Contributors CC and SK have written the first manuscript for this trial and they will contribute to monitoring this trial. YK and MK have contributed to the development of the protocol. BHJ and YCS have edited the first manuscript. SGK has conducted all the procedures for this protocol. All authors have read and approved the final manuscript.

Funding This work was supported by a grant of the Korea Health Technology R\&D Project through the Korea Health Industry Development Institute (KHIDI), funded by the Ministry of Health \& Welfare, Republic of Korea (grant number HI12C1889). The management, analysis and reporting of study will be conducted independently by the study investigators.

Competing interests None declared.

Patient consent Obtained.

Ethics approval The Institutional Review Board of the Catholic Kwandong University International St. Mary's Hospital approved the study (reference IS16MNSI0011).

Provenance and peer review Not commissioned; externally peer reviewed.

Open access This is an open access article distributed in accordance with the Creative Commons Attribution Non Commercial (CC BY-NC 4.0) license, which permits others to distribute, remix, adapt, build upon this work non-commercially, and license their derivative works on different terms, provided the original work is properly cited and the use is non-commercial. See: http://creativecommons.org/ licenses/by-nc/4.0/

(c) Article author(s) (or their employer(s) unless otherwise stated in the text of the article) 2018. All rights reserved. No commercial use is permitted unless otherwise expressly granted.

\section{REFERENCES}

1. Berger AM, Gerber LH, Mayer DK. Cancer-related fatigue: implications for breast cancer survivors. Cancer 2012;118(8 Suppl):2261-9.

2. Berger AM, Mooney K, Alvarez-Perez A, et al. Cancer-Related Fatigue, Version 2.2015. J Natl Compr Canc Netw 2015;13:1012-39.

3. de Jong N, Candel MJ, Schouten HC, et al. Prevalence and course of fatigue in breast cancer patients receiving adjuvant chemotherapy. Ann Oncol 2004;15:896-905.

4. Sitzia J, Huggins L. Side effects of cyclophosphamide, methotrexate, and 5-fluorouracil (CMF) chemotherapy for breast cancer. Cancer Pract 1998;6:13-21.

5. Jacobsen PB, Hann DM, Azzarello LM, et al. Fatigue in women receiving adjuvant chemotherapy for breast cancer: characteristics, course, and correlates. J Pain Symptom Manage 1999;18:233-42.

6. Song DS, Yeo CD, Park JM, et al. The prevalence of fatigue in cancer patients at St. Vincent's hospital. Korean journal of internal medicine 2007;73:512-8.

7. Curt GA. The Impact of Fatigue on Patients with Cancer: Overview of FATIGUE 1 and 2. Oncologist 2000;5(Suppl 2):9-12.

8. Given B. Cancer-related fatigue: a brief overview of current nursing perspectives and experiences. Clin J Oncol Nurs 2008;12(5 Suppl):7-9.

9. Gutstein HB. The biologic basis of fatigue. Cancer $2001 ; 92(6$ Suppl):1678-83.

10. Barsevick AM, Newhall T, Brown S. Management of cancer-related fatigue. Clin J Oncol Nurs 2008;12(5 Suppl):21-5.

11. KFDA. Research on Intake of Chinese Medicine by Korean: KFDA, 2007:36-9.

12. Oh S, Cheon C, Park S, et al. The Analysis of the Recent Research Trend of Sipjeondabo-tang in Korea. J Soc Prev Korean 2014;18:113-23.

13. Ministry of Food and Drug Safety. Online medicine library (drug.mfds. go.kr): Ministry of Food and Drug Safety. http://drug.mfds.go.kr/html/ bxsSearchDrugProduct_dsie.jsp?item_Seq=197900047 (accessed 11 Dec 2017).

14. Huang SM, Chien LY, Tai CJ, et al. Effectiveness of 3-week intervention of Shi Quan Da Bu Tang for alleviating hematotoxicity 
among patients with breast carcinoma receiving chemotherapy. Integr Cancer Ther 2013;12:136-44.

15. Nakamoto H, Mimura T, Honda N. Orally administrated Juzen-taihoto/TJ-48 ameliorates erythropoietin (rHuEPO)-resistant anemia in patients on hemodialysis. Hemodial Int 2008;12(Suppl 2):S9-S14.

16. Kishida $Y$, Nishii $T$, Inoue $T$, et al. Juzentaihoto (TJ-48), a traditional Japanese herbal medicine, influences hemoglobin recovery during preoperative autologous blood donation and after hip surgery. Int $J$ Clin Pharmacol Ther 2009;47:716-21.

17. Julious SA. Sample size of 12 per group rule of thumb for a pilot study. Pharm Stat 2005;4:287-91.

18. Sim J, Lewis M. The size of a pilot study for a clinical trial should be calculated in relation to considerations of precision and efficiency. $J$ Clin Epidemiol 2012;65:301-8.

19. Ryu BH, Kim JS, Kim JS, et al. Bojungikki-tang for cancer-related fatigue: a pilot randomized clinical trial. Integr Cancer Ther 2010;9:331-8.

20. Xu Y, Chen Y, Li P, et al. Ren Shen Yangrong Tang for Fatigue in Cancer Survivors: A Phase I/II Open-Label Study. J Altern Complement Med 2015;21:281-7.

21. Mendoza TR, Wang XS, Cleeland CS, et al. The rapid assessment of fatigue severity in cancer patients: use of the Brief Fatigue Inventory. Cancer 1999:85:1186-96.

22. Yun YH, Lee MK, Chun HN, et al. Fatigue in the general Korean population: application and normative data of the Brief Fatigue Inventory. J Pain Symptom Manage 2008;36:259-67.

23. Chang YJ, Lee JS, Lee CG, et al. Assessment of clinical relevant fatigue level in cancer. Support Care Cancer 2007;15:891-6.

24. Aaronson NK, Ahmedzai S, Bergman B, et al. The European Organization for Research and Treatment of Cancer QLQ-C30: a quality-of-life instrument for use in international clinical trials in oncology. J Natl Cancer Inst 1993;85:365-76.

25. Knobel H, Loge JH, Brenne E, et al. The validity of EORTC QLQ-C30 fatigue scale in advanced cancer patients and cancer survivors. Palliat Med 2003;17:664-72.

26. Minton O, Stone P. A systematic review of the scales used for the measurement of cancer-related fatigue (CRF). Ann Oncol 2009;20:17-25

27. Yun YH, Park YS, Lee ES, et al. Validation of the Korean version of the EORTC QLQ-C30. Qual Life Res 2004;13:863-8.

28. Yun YH, Bae SH, Kang IO, et al. Cross-cultural application of the Korean version of the European Organization for Research and Treatment of Cancer (EORTC) Breast-Cancer-Specific Quality of Life Questionnaire (EORTC QLQ-BR23). Support Care Cancer 2004;12:441-5.

29. U.S.Department of Health and Human Services. Common Terminology Criteria for Adverse Events (CTCAE) v4.03: National Institutes of Health National Cancer Institute, 2010.

30. Ishikawa S, Ishikawa T, Tezuka C, et al. Efficacy of Juzentaihoto for Tumor Immunotherapy in B16 Melanoma Metastasis Model. Evid Based Complement Alternat Med 2017;2017:1-8.

31. Ishikawa S, Ishikawa T, Asano K, et al. Suppressive effect of juzentaihoto on vascularization induced by b16 melanoma cells in vitro and in vivo. Evid Based Complement Alternat Med 2012;2012:1-9.

32. Choi YK, Jung KY, Woo SM, et al. Effect of Sipjeondaebo-tang on cancer-induced anorexia and cachexia in CT-26 tumor-bearing mice. Mediators Inflamm 2014;2014:1-10.

33. Ogawa K, Omatsu T, Matsumoto C, et al. Protective effect of the Japanese traditional medicine juzentaihoto on myelosuppression induced by the anticancer drug TS-1 and identification of a potential biomarker of this effect. BMC Complement Altern Med 2012;12:118.

34. Kamiyama H, Takano S, Ishikawa E, et al. Anti-angiogenic and immunomodulatory effect of the herbal medicine "Juzen-taiho-to" on malignant glioma. Biol Pharm Bull 2005;28:2111-6.

35. Tagami K, Niwa K, Lian Z, et al. Preventive effect of Juzen-taiho-to on endometrial carcinogenesis in mice is based on Shimotsu-to constituent. Biol Pharm Bull 2004;27:156-61.

36. Nishiuchi T, Okutani $Y$, Yamagishi $Y$, et al. Synergistic effect between Juzen-taiho-to, a Japanese traditional herbal medicine, and gemcitabine single-agent chemotherapy for advanced biliary tract cancer. J Altern Complement Med 2013;19:593-7.

37. Yoshioka H, Fukaya S, Miura N, et al. Suppressive Effect of Kampo Formula "Juzen-taiho-to" on Carbon Tetrachloride-Induced Hepatotoxicity in Mice. Biol Pharm Bull 2016;39:1564-7.

38. Jeon WY, Shin IS, Shin HK, et al. Gastroprotective effect of the traditional herbal medicine, Sipjeondaebo-tang water extract, against ethanol-induced gastric mucosal injury. BMC Complement Altern Med 2014;14:373.

39. Lee YM, Kim CS, Sohn E, et al. Sipjeondaebo-tang, a traditional herbal formula, inhibits retinal neovascularization in a mouse model of oxygen-induced retinopathy. Tohoku J Exp Med 2014;234:229-36.

40. Takeda O, Toyama T, Watanabe K, et al. Ameliorating effects of Juzentaihoto on restraint stress and P. gingivalis-induced alveolar bone loss. Arch Oral Biol 2014;59:1130-8.

41. Hara $\mathrm{H}$, Kataoka S, Anan M, et al. The therapeutic effects of the herbal medicine, Juzen-taiho-to, on amyloid-beta burden in a mouse model of Alzheimer's disease. J Alzheimers Dis 2010;20:427-39.

42. Ishiura $Y$, Shiba Y, Terasaki Y, et al. [Effect of Japanese Traditional Medicine, TJ-48, on the Quality of Life of Patients with Non-Small Cell Lung Cancer Receiving Outpatient Chemotherapy]. Gan To Kagaku Ryoho 2016;43:331-4.

43. Cheon C, Park S, Park YL, et al. Sipjeondaebo-tang in patients with cancer with anorexia: a protocol for a pilot, randomised, controlled trial. BMJ Open 2016;6:e011212.

44. Ikemoto T, Shimada M, Iwahashi S, et al. Changes of immunological parameters with administration of Japanese Kampo medicine (Juzen-Taihoto/TJ-48) in patients with advanced pancreatic cancer. Int J Clin Oncol 2014;19:81-6.

45. Barton DL, Soori GS, Bauer BA, et al. Pilot study of Panax quinquefolius (American ginseng) to improve cancer-related fatigue: a randomized, double-blind, dose-finding evaluation: NCCTG trial N03CA. Support Care Cancer 2010;18:179-87.

46. Barton DL, Liu H, Dakhil SR, et al. Wisconsin Ginseng (Panax quinquefolius) to improve cancer-related fatigue: a randomized, double-blind trial, N07C2. J Natl Cancer Inst 2013;105:1230-8.

47. Lee JY, Oh HK, Ryu HS, et al. Efficacy and Safety of the Traditional Herbal Medicine, Gamiguibi-tang, in Patients With Cancer-Related Sleep Disturbance: A Prospective, Randomized, Wait-List-Controlled, Pilot Study. Integr Cancer Ther 2018;17:1534735417734914.

48. Jeong JS, Ryu BH, Kim JS, et al. Bojungikki-tang for cancerrelated fatigue: a pilot randomized clinical trial. Integr Cancer Ther 2010;9:331-8.

49. Lee J, Yoon SW. Efficacy and Safety of Moxibustion for Relieving Pain in Patients With Metastatic Cancer: A Pilot, Randomized, Single-Blind, Sham-Controlled Trial. Integr Cancer Ther 2014;13:211-6

50. Cheon C, Yoo JE, Yoo HS, et al. Efficacy and Safety of Sipjeondaebo-Tang for Anorexia in Patients with Cancer: A Pilot, Randomized, Double-Blind, Placebo-Controlled Trial. Evid Based Complement Alternat Med 2017;2017:1-9. 